\section{Eine Methode zum direkten Nachweis von Leitfähigkeitsinhomogenitäten an Korngrenzen}

\author{
Von P. Gerthsen und K. H. Härdtu
}

Philips Zentrallaboratorium GmbH., Laboratorium Aachen (Z. Naturforschg. 18 a, 423-424 [1963] ; eingegangen am 25. Januar 1963)

Die Korngrenzen in polykristallinen Halbleitern vornehmlich keramisch hergestellten Oxyden - sind vielfach Bereiche erhöhten elektrischen Widerstandes. Der Widerstand eines Körpers ist hier bestimmt durch die Serienschaltung der Widerstände des Korninnnern (Volumwiderstand der Substanz) und der erhöhten Widerstände dünner Schichten an den Korngrenzen. Abb. 1 zeigt das eindimensionale Modell ${ }^{1}$ eines polykristallinen Leiters mit einer Folge von Körnern (2), die durch hochohmige Randschichten kleiner Dicke (1) an den Korngrenzen unterbrochen ist.

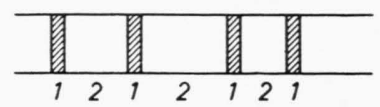

Abb. 1. Modell eines polykristallinen Leiters. 1: hochohmige Randschichten an den Korngrenzen, 2: Körner.

Ein Nachweis der Existenz dieser hochohmigen Randschichten konnte für keramisches Material mit den üblichen Methoden der Widerstandsmessung infolge der geringen Korngrößen nicht erfolgen. Die Existenz der hochohmigen Schichten wird hier gefolgert aus dem Wechselstromverhalten der elektrischen Leitfähigkeit und der Dielektrizitätskonstanten ${ }^{2}$. Hier zeigt die gemessene Frequenzabhängigkeit von Widerstand und Kapazität bzw. der Dielektrizitätskonstanten, gemessen in einer Brückenschaltung nach Abb. 2 a, Dispersionsverteilungen. Diese Dispersionsverteilungen des Widerstandes sind charakteristisch für eine in Abb. 2 b dargestellte Kombination von Widerständen und Kapazitäten. Abb. 2 b wiederum ist das Ersatzschaltbild ${ }^{1}$ eines

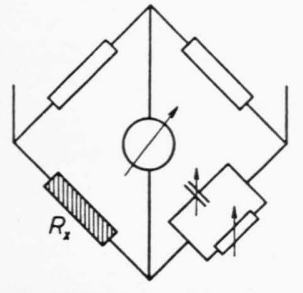

Abb. 2 a.

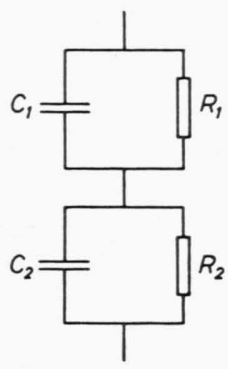

Abb. 2 b.
Abb. 2 a. Schaltbild einer Brücke zur Messung des Widerstandes $R_{x}$ einer polykristallinen Probe.

Abb. 2 b. Ersatzschaltbild von Korn und Korngrenze.

1 C. G. Koops, Phys. Rev. 83, 121 [1951].

2 Zusammenfassende Darstellung und Literaturhinweise in: Progress in Semiconductors, Vol. 4, Beitrag J. Volger, p. 207, Heywood \& Company Ltd., London 1960.
Korns vom Widerstand $R_{2}$ und seiner parallel geschalteten Kapazität $C_{2}$, welchem seine Korngrenze wiederum bestehend aus der Parallelschaltung von Korngrenzenwiderstand $R_{1}$ und Korngrenzenkapazität $C_{1}-$ in Reihe geschaltet ist.

Ein Beispiel für die Existenz hochohmiger Schichten an den Korngrenzen ist halbleitendes $\mathrm{BaTiO}_{3}{ }^{3,4}$. Abb. 3 zeigt die gemessene Frequenzabhängigkeit des spezifischen Widerstandes mit der charakteristischen Dispersionsverteilung.

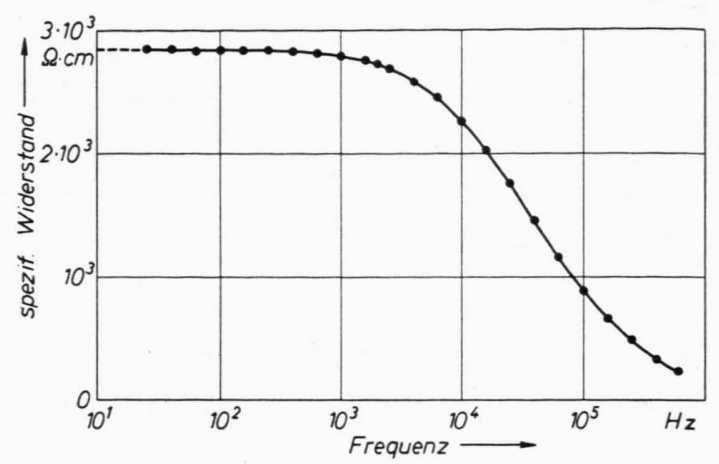

Abb. 3. Frequenzabhängigkeit des spezifischen Widerstandes von polykristallinem $\mathrm{BaTiO}_{3}$, dotiert mit 3 Atom-Promille La.

Die im folgenden beschriebene Methode zur Sichtbarmachung der Korngrenzenwiderstände macht Gebrauch von der erhöhten Feldstärke in der vom elektrischen Strom durchflossenen Randschicht. Dieses starke Feld streut - nach Art der Randstörung des Plattenkondensators - aus den Durchstoßlinien der Korngrenzen durch die Oberfläche. Dielektrische Partikelchen erfahren durch dieses stark inhomogene Streufeld eine Kraft in Richtung auf die Korngrenze. Schichtet man daher auf die Oberfläche des von einem Strom durchflossenen polykristallinen Leiters eine Suspension dielektrischer Teilchen in einer Flüssigkeit, so erwartet man eine bevorzugte Ansammlung der Teilchen an den Korngrenzen, falls die Dielektrizitätskonstante der Partikel groß ist gegen diejenige der Flüssigkeit. Diese Markierung sollte im Mikroskop erkennbar sein.

Von den Kräften des Streufeldes auf die Partikelchen erwarten wir, daß sie hinreichend stark sind im Vergleich zu denjenigen Kräften, die einen räumlichen Konzentrationsausgleich der Teilchen erstreben (thermische Schwankungen und Reibungskräfte infolge von Strömungen). Zur größenordnungsmäßigen Abschätzung der Feldkräfte auf das Teilchen gelangt man folgendermaßen: Auf das Teilchen mit Radius $a$ - Dielektrizitätskonstante des Partikelchens groß gegen diejenige der Flüssigkeit - wirkt die Kraft vom Betrage

$$
|K|=\frac{1}{2} a^{3} \operatorname{grad}\left(E^{2}\right) \text {. }
$$

3 P. W. Haayman, R. W. Dam u. H. A. Klasens, DBP 929350 [1955].

4 W. Heywang, Solid State Electronics 3, 51 [1961]. 
Die Feldlinien nehmen wir in hinreichender Entfernung von der Korngrenze als kreisförmig an. Die Mittelpunkte der Kreise liegen auf den Durchstoßlinien der Korngrenzen durch die Oberfläche.

$$
|E| \approx \frac{U}{\pi r} \text { und }\left|\operatorname{grad}\left(E^{2}\right)\right| \approx \frac{2}{\pi^{2}} \frac{U^{2}}{r^{3}}
$$

Hierbei bedeutet $U$ die an der Korngrenze abfallende Spannung. Für die Kraft, mit der das Teilchen auf der Oberfläche festgehalten wird, erwarten wir damit die Größenordnung

$$
|K| \approx \frac{1}{2} a^{3} \frac{2 U^{2}}{\pi^{2} a^{3}}=\frac{1}{\pi^{2}} U^{2},
$$

welche offenbar unabhängig von der Größe des Teilchens und proportional dem Quadrat der Spannung ist.
Demnach ist das Teilchen bei Spannungen von 1 Volt pro Korngrenze mit größenordnungsmäßig $10^{-6}$ dyn an eine Korngrenze gebunden. Diese Kräfte sind derart groß, daß bei Teilchengrößen von ca. $1 \mu$ der Versuch ohne wesentliche Vorsichtsmaßregeln durchführbar ist.

Abb. 4a* zeigt das kristallographische Schliffbild einer keramischen $\mathrm{BaTiO}_{3}$-Probe, deren Frequenzabhängigkeit des elektrischen Widerstandes in Abb. 3 wiedergegeben ist. Die Korngrenzen sind deutlich erkennbar. Abb. 4 b zeigt dieselbe Oberfläche mit überschichteter Suspension von $\mathrm{TiO}_{2}$-Pulver in Silicon-Öl im stromdurchflossenen Zustand. Die Anhäufung der Teilchen an den Korngrenzen ist deutlich erkennbar.

*Abb. 4 a, b auf Tafel S. 412 b.

\section{Oszillatorische magnetische Widerstandsänderung an Wismut-Tellurid bei tiefen Temperaturen}

\section{Von K. Auch und G. Landwehr}

Physikalisch-Technische Bundesanstalt, Braunschweig und Institut für Technische Physik der Technischen Hochschule Braunschweig

(Z. Naturforschg. 18 a, 424-427 [1963] ; eingegangen am 7. Februar 1963)

Nach Analyse von galvanomagnetischen Messungen bei $77^{\circ} \mathrm{K}$ wurde von Drabble ${ }^{1}$ ein EnergiebänderModell für p- $\mathrm{Bi}_{2} \mathrm{Te}_{3}$ vorgeschlagen, bei dem die Flächen konstanter Energie im $\boldsymbol{k}$-Raum ( $\boldsymbol{k}=$ Wellenzahlvektor) Ellipsoide sind, deren Zentren in den Spiegelebenen des rhomboedrischen Gitters liegen. $\left(\mathrm{Bi}_{2} \mathrm{Te}_{3}\right.$ gehört zur Kristallklasse R $\overline{3} \mathrm{~m}$.) Dieses Modell wurde im wesentlichen von Efimova ${ }^{2}$ et al. bestätigt, die galvanomagnetische Untersuchungen an $\mathrm{Bi}_{2} \mathrm{Te}_{3} \mathrm{zu}$ Helium'Temperaturen ausdehnten. Eine Entscheidung darüber, ob 6 Ellipsoide vorliegen oder nur 3, deren Zentren auf den Begrenzungsebenen der Brillouin-Zone liegen, konnte jedoch nicht getroffen werden. Eine Inspektion der publizierten Daten zeigt, daß es möglich sein sollte, diese Frage zu klären, und zwar durch Messungen der magnetischen Widerstandsänderung von hinreichend reinen Einkristallen in starken Magnetfeldern bei tiefen Temperaturen. Unter diesen Umständen sollten oszillatorische Effekte in den Transportgrößen beobachtbar sein. Damit sind Erscheinungen gemeint, die auf der gleichen Grundlage beruhen, wie der bekannte DE HAAsvan Alphen-Effekt ${ }^{3}$. In einem Magnetfeld wird die Bewegung eines quasifreien Kristallelektrons senkrecht zur Feldrichtung gequantelt, es treten diskrete Energieniveaus auf, die oftmals als LANDAU-Niveaus ${ }^{4}$ bezeichnet werden. Die erlaubten Energiewerte haben (bei Ver-

1 J. R. Drabble, Proc. Phys. Soc., Lond. 72, 380 [1958].

2 B. A. Efimova, I. Y. Korenblum, V. I. Novikov u. A. G. Ostroumov, Soviet Phys.-Solid State 3, 2004 [1962]. nachlässigung des Spins) im isotropen Fall die Form

$E_{n}=\left(n+\frac{1}{2}\right) \hbar \omega+\frac{\hbar^{2}}{2 m^{*}} k_{z}^{2} \quad(n=0,1,2, \ldots)$,

wenn das Magnetfeld in $z$-Richtung weist. Es bedeuten: $\omega=e H / m^{*} c=$ Zyklotronfrequenz, mit $e=$ Elementarladung, $H=$ magnetische Feldstärke, $m^{*}=$ effektive Masse des Kristallelektrons, $c=$ Lichtgeschwindigkeit, $k_{z}=z$-Komponente des Wellenzahlvektors. Die Dichte der besetzbaren Zustände wächst nicht mehr quasi-kontinuierlich mit zunehmender Energie wie im feldfreien Fall, sondern hat ausgeprägte Maxima bei den Energien der Landau-Niveaus. Oszillationen in den Transporterscheinungen (oder der Suszeptibilität) als Funktion der magnetischen Feldstärke können bei einem entarteten Halbleiter unter folgenden Bedingungen auftreten: a) $\omega \tau>1, \quad$ b) $\hbar \omega>k T$, c) $\hbar \omega<\zeta$ mit $\tau=$ Stoßzeit, $\zeta=$ Fermi-Energie. Die mittlere freie Weglänge der Elektronen muß so groß sein, daß mindestens ein ungestörter Umlauf im Magnetfeld zustande kommt. Die diskrete Natur der Energieniveaus darf nicht durch $\mathrm{zu}$ hohe Temperaturen verwischt werden, und es ist weiterhin zu fordern, daß für technisch herstellbare Magnetfelder und nicht zu große Werte von $n$ die Bedingung:

$$
\left(n+\frac{1}{2}\right) \hbar \omega=\zeta
$$

erfüllt werden kann. Sobald mit wachsendem Magnetfeld $H$ die Energie eines Landau-Niveaus mit der Fermi-Energie zusammenfällt, hat die Zustandsdichte ein Maximum. Als Folge davon tritt in einem longitudinalen Magnetfeld ein Minimum des elektrischen Widerstandes auf. Bei Variation von $H$ werden Oszillationen in den Transporterscheinungen beobachtet, deren Periode $H^{-1}$ proportional ist. Oszillationen in der magnetischen Widerstandsänderung wurden zum erstenmal

3 W. J. de Haas u. P. M. van Alphen, Leiden Comm. 208 d, 212 a [1930].

${ }^{4}$ L. D. Landau, Z. Phys. 64, 629 [1930]. 